\section{Zur Frage der Ausgestaltung des chemischen Schul- und Hochschulunterrichts.}

\author{
Von Prof. Dr. H. Grossmann, Berlin. \\ (Eingeg. 5.18. 1917.)
}

Zu den in England gegen Deutschland und seine geistigen Lehrer außerordentlich häufig erhobenen, gänzlich unberechtigten Vorwürfen gehört auch der zu einem reinen Schlagwort der Tagespresse gewordene Hinweis, daß der deutsche Unterricht nicht genügend Wert auf Ethik und Charakterbildung lege und nur darauf hinarbeite, tatsăchliohes Wissen zu verbreiten. Aus dieser fehlerhaften Gestaltung des Unterrichts, so wird dann gern weiter geschlossen, erklären sich auch in letzter Linie jene furchtbaren Ausschreitungen im Kriege, die man einseitig dem deutschen Heere zuschreibt, und von deren Wahrheit der englisohe Philister auf Grund der amtlichen "Greuelveröffentlichungen" meist ohne jede Kritik fest überzeugt ist. Als leuohtendes Gegenbild steht demgegenüber natürlich dann die alte englische Erziehung da, die den humanistischen Wissenschaften den breitesten Raum gewährt und ohne weiteres "Humanistik" mit „Humanitat" als gleichbedeutend erklärt. Daß nicht die schlechtesten Köpfe Englands an dieser übertrieben humanistisohen Erziehung der höheren Gesellschaftsklassen zu wiederholten Malen begründete Zweifel geäußert haben, wird dabei allerdings nach Möglichkeit übersehen. Aus der zweifellos festatehenden Tatsache, daß auch die auf den höheren Sohulen Englands ausgebildeten jungen und älteren Soldaten sich vorzüglich geschlagen haben, wie auch die deutsohe Heeresleitung stets anerkannt hat, schließt man aber weiter in vielen Fällen, daß diese Bewährung im Kampfe in unmittelbarem Zusammenhang mit dem humanistischen Unterricht stehe, und erklärt infolgedessen eine jede Reform des Unterrichts unter Erweiterung des den Naturwissenschaften gewidmeten Spielraums für überflüssig und schädlich. Fast die gleichen Argumente führen übrigens auch die ziemlich zahlreichen und durchaus einseitigen Anhänger des humanistischen Unterrichts in Deutschland gegenüber den neueren Forderungen zugunsten der Naturwissensohaften ins Feld. Während man aber auf der Seite dieser Gegner des naturwissenschaftlichen Unterrichts auf den deutschen Schulen gerade in der letzten Zeit eine sehr eifrige Tätigkeit bemerken kann, wio sich auch aus den zahlreichen Protesterklärungen angesehener Hoohsohullehrer an verschiedenen Universitäten gegen die zeitgemäße Ausdehnung des naturwissenschaftlichen Unterrichts ergibt, haben sich die Freunde der Naturwissensohaften in Deutschland in der Offentlichkeit bisher leider viel weniger gerührt. Das ist zweifellos sehr zu bedauern, denn wenn auch die Gegner auf die Dauer einen aussichtslosen Kampf ausfechten, so kann eine Untaitigkeit der Freunde des naturwissenschaftlichen Unterrichts oder auch schon ein gewisses Nachlassen der Propagandatätigkeit leicht dazu führen, daß die berechtigten Forderungen der Gegenwart in Deutsohland erst weit spater verwirklicht werden, als es im Interesse der Gesamtheit zu wünschen würe. Man vergesse aber dooh niobt, daBdienachsten Jahrenaohdem Kriege a uch fürdieganze Zukunft des deutschen Volkes vonder gröbten Bedeutung sein werden, und da B oine planmäBige Reform des Unterriohts, dieauch den Naturwissenschaften ihr Reoht labt, sebr viel wird dazubeitragen können, die unendliohen Schädendes Weltkriegeswe. nigstens zum Teil im La ufe der näohsten Zeit allmählioh zu mildern.

Es war daher ein außerordentlich guter Gedanke des Vereins zur Förderung des mathematischen und naturwissenschaftlichen Unterrichts, im Mai 1915 einen Preisbewerb über die folgende Frage auszuschreiben:

Welehe Forderungen sind naoh dem Kriege andie Erziehung der deatsohen Jugendzustel. len, und waskanndermathematische und naturwissensohaftliohe Unterricht zur Verwirklichung dieser Frage beitragen?

Auf dieses Preisausschreiben sind neuerdings von der aus den Herren Friedrioh Poske (Vorsitzender des Vereins), R. von Hanstein, W. Lietzmann, B. Sohmidt und H. Timerding bestehenden Kommission zwei Arbeiten als besonders hervorragend bezeichnet worden. Den ersten Preis errang die der Mathematik gewidmete Arbeit von Dr. W. Sch miedeberg, Oberlehrer an der Oberrealschule zu Bielefeld, der zweite Preis wurde dagegen Dr. Ge org Klatt, Professor an der Oberrealschule zu Görlitz für seine Arbeit über den chemischen und biologischen Unterricht zugesprochen. Auf besondere Aufforderung des Vereins hat dann noch Dr. Georg Wetzste in, Professor am Realgymnasium zu Augsburg, seine Gedanken über den physikalischen Unterricht zusammengefa $ß t$, und diese drei Arbeiten sind neuerdings gemeinsam unter dem Titel: „Die Bedeutung des mathematio he n und naturwissensohaftlichen Unterriohts für die Erziehung der Jugend," in einem 262 Seiten starken Bande im Verlage von Otto Salle, Berlin, $(4,50 \mathrm{M})$ veröffentlicht worden.

Wenn nun an dieser Stelle auf einzelne Darlegungen von Prof. $K$ l a t $t$ besonders hingewiesen wird, so geschieht das, weil seine Arbeit es in der Tat verdient, nicht nur in Lehrerkreisen gelesen und beachtet zu werden, sondern weil auch die Gefahr besteht, daß eine so vorzügliche und klare Darstellung in der heutigen Zeit selbst von denjenigen üborsehen wird, die den ohemischen Unterrichtsfragen auf den deutschen Schulen und Hochschulen ein besonderes Interesse zuwenden.

Als die erste Aufgabe der Naturwissenschaften bezeichnet es der Verfasser mit Recht, den $S$ in $n$ fü $r$ die Wirklichkeit zu weoken und nicht nurdas Wort, sondernauch die Ansobaung zu pflegen. Wenn nun aber auch in den Lehrplänen von 1901 möglichste Anschaulichkeit und ausgiebigste Versuche empfohlen werden, so erscheint doch der Erfolg aller dieser Bemühungen dadurch sehr in Frage gestellt, daB die Naturwissenschaften innerhalb der gesamten Unterrichtsfächer an den höheren Sohulen im deutschen Reich meist einen zu kleinen Raum einnehmen.

In dem Tatsachencharakter, den der Jnhalt des naturwissenschaftlichen Wissens trägt, hat man in der Tat einen gewaltigen, heute bei weitem noch nicht ausgenutzten Wert zu erblicken. Ganz richtig wird auch hervorgehoben, daB das schärfste Denken, das wärmste Fühlen und das ernsthafteste Wollen zur Phantasterei führen muß, wenn es nicht von dem Boden der Tatsachen seinen Ausgang nimmt. Man denkt unwillkürlich bei diesen Darlegungen an die Zeiten der Naturphilosophie, deren Sohwächen seinerzeit besonders $\mathrm{J}$ u s $\mathrm{t}$ u s $\mathrm{L}$ i $\mathrm{e}$ big mit scharfer, aber durchaus berechtigter Kritik gegeiBelt hat. Daß Prof. K I a t t nicht zu der Zahl derjenigen deutschen Oberlehrer gehört, denen niemals ein Zweifel an ihrer Gottähnlichkeit kommt, zeigt einmal sein Hinweis auf den Tatsachensinn als eine echt deutsche Tugend, „womit allerdings nicht gesagt sein soll, da $B$ wir diese Tugend bereits im vollsten $\mathrm{Ma}$ Be besitzen, als vielmehr, daß sie in der Richtung unseres Ideals liegt". Dann ist aber auch sein aufrichtiges und praktisch erprobtes Streben rühmend hervorzuheben, den Schülern im chemischen Unterricht als Freund und Berater und nicht nur als drohender Selbstbeherrsoher zur Seite zu treten. Er erwähnt z. B. einen Versuch, wobei die Primaner sich schriftlich darüber äußern mußten, welche Ziele ihrer Meinung nach mit dem chemischen Praktikum auf der Schule verfolgt werden. Es ergab sioh hierbei mit aller Dentlichkeit, daß auch die Schüler schon für die Absicht, sio zum Nachdenken über den Wert ihrer Arbeit zu veranlassen, durchaus empfänglich waren. Auch die Veranstaltang von „,naturwissenschaftlichen Kreisen", wie sie der Verfasser in Görlitz eingerichtet hat, könnte sicherlich viel dazu beitragen, das naturwissenschaftliche Interesse schon auf den Schulen zu fördern und dabei auoh eine erzieherische Wirkung auszü̈ben. Mit vollem Recht wird ferner gegenüber der vielfach verbreiteten Ansicht, daB die durch die Naturwissenschaften gesteigerte Beobachtungsfäbigkeit zwar für den künftigen Mediziner und Naturforscher, dagegen nicht für den Juristen und Altphilologen notwendig sei, geltend gemacht, „da $\beta$ man in $Z$ ukunt ganze Menschen nötig baben werde, und zwarnicht weniger bei den Verwal. tungsbehörden, als unterden Ärzten und Teohnikurn".

Die neuerdings von verschiedenen Seiten begünstigten Versuche, den Referendaren eine "praktisch" Ausbildung zu geben, indem man ihnen Einblick in Fabriken und ähnliohen Betrieben gewährt, bezeichnet $\mathrm{KI}$ a t $\mathrm{t}$ als eine etwas spät einsetzende Maßnahme, der es kaum gelingen dürfte, eine Bresche in die Mauer zu legen, die der Bu c h betri e b in Schule und Universität in den Köpfen der jungen Menschen aufgerichtet hat. (S. 180.)

Es fragt sich nun aber doch, ob der verehrte Verfasser mit solchen Ausführungen den Universitäten nicht etwas unrecht tut. An verschiedenen Stellen, z. B. in $L$ e i p z g, T ü b i n g e n, E r langen, Berlin usw., von den technischen Hochschulen und den Handelshochschulen gar nicht zu reden, bemüht man sich doch 
schon seit längerer Zeit, ähnliche Bestrebungen, die durch den Weltkrieg zweifellos auch besonders gefördert werden dürften, durchzusetzen und besonders der Chemie das Verständnis weiter Kreise zu erschließen. Der Bromberger Oberlehrer $I_{\mathbf{L}}$ Trink w a 1 t e $r$ hat in einem sehr beachtenswerten Aufsatz über: ,F r a g e n des chemischen Unterrichts" in der Zeitschrift: „Aus der Natur" (1917, S. 380-387 und 456-463) nicht mit Unrecht darauf hingewiesen, da $B$ man auch die führenden Geister der Chemio nicht ganz von den Vorwurf freispreohen könne, daß sie sich zu wenig bemühten, weitere Kreise für die Bedeutung chemischer Forschung zu interessieren; und auch seine weitere Bomerkung, $\mathrm{daB}$ die Vertreter anderer Wissenschaften und der Kunst den Chemikern auf dem Gebiet einer gesunden Popularisierung der Wissenschaft weit über seien, entbehrt durchaus nicht einer gewissen Berechtigung. Andererseits darf man aber auch nicht verkennen, $\mathrm{da} B$ in neuerer Zeit das Interesse für die Chemie in größeren Kreisen etwas gewachsen ist. Allerdings gibt es auch heute noch manche Chemiker, die allen solohen Bestrebungen mit einem gewissen Mißtrauen gegenüberstehen, weil sie daraus eine Gefährdung des rein wissenschaftliohen Charakters der Chemie befürchten.

Was Prof. K la t $t$ über die Bedeutung der Naturwissenschaften und besonders der Chemie für die staatsbürgerliche Erziehung sagt, die nach seinen Wünschen schon auf der Schule einsetzen sollte, enthält ebenfalls eine ganze Reihe von Anregungen, denen auch derjenige seine Zustimmung nicht versagen wird, der eine allgemeine Einführung in die Volkswirtschaft weniger der Sohule als den Hoohschulen zur Aufgabe stellen möchte. Ganz richtig wird allerdings hervorgehoben, daß die großen Mächte unserer Volkswirtschaft, Landwirtschaft und Industrie, ihre Bedeutung, ihre Bedürfnisse und ihre gegenseitigen Abhängigkeitsverhältnisse nur derjenige verstehen kann, der über einige naturwissenschaftliche Kenntnisse verfügt. ,Eine Durohdringung der ohemisch-technischen Tatsachen mit volkswirtschaftlichen Gesichtspunkten, das ist in der Tat das Ideal, dem auch der Technologe wird nachstreben müssen, der im Unterricht auf der Hochsohule auch auf die Angehörigen anderer Berufe wird einwirken wollen." Bis jetzt ist ein Versuch, die Volkswirtschaft im naturkundlichen und chemischen Unterricht darzustellen, noch nicht in befriedigender Weise gemacht worden. Es ist aber sehr beachtenswert, daB jetzt auch aus den Kreisen der Lehrerschaft heraus der Wunsch geäußert wird, eine solche Anleitung zu erbalten.

Man wird in der Tat unter Berüoksiohtigung der leider feststehenden Tatsache, daB auch im deutschen Volk die Kenntnisse von den Errungensohaften der Technik und der Industrie nooh viel zu wenig verbreitet sind, immer wieder darauf aufmerksam machen müssen, daß es, wie Klatt sehr richtig sagt, $n$ i c b t di e ro be Kraft der Arbeitsfäuteallein ist, dieDeutsoblands Industrie geschaffen hat, sonderndaB es vor allem geistige Krätegewesensind, denen man den Aufsohwung von Handel und Industrie z u verdanke $n$ at. Nicht nur die jetzt so beliebte Chemie des Krieges gilt es kennen zu lernen, sondern den Versuch zu machen, dem Sohüler und Studenten mit Hilfe einiger passend ausgewählter Beispiele aus der Geschichte der ohemischen Industrie einen Einbliok in dieses gewaltige Gebiet nationaler Arbeit zu verschaffen. Wenn man dadurch zu dem Ergebnis gelangt, Deutschlands Stellung in der Weltwirtsohaft frei von chauvinistisohen Vorurteilen allmählich zu begreifen, so bedeutet das in der Tat auoh ein Stück nationaler Erziehung.

Herr Prof. K la t t, der mit seinen Schülern von Görlitz aus vielfach technische Betriebe besichtigt hat, hält es auch für notwendig, bei der vorherigen Erörterung dieser Besichtigungen das soziale Moment nicht zu vergessen. Warmherziges soziales Empfinden $\mathrm{zu}$ wecken, ist sicherlich ebenfalls eine vaterländisch wertvolle Arbeit, die in den obersten Klessen, aber wohl besser noch auf den Universitäten und technischen Hochschulen, wie auch auf den Handelshoohschulen, niemals vergessen werden sollte, da doch gerade die deutsche Sozialpolitik den anderen Völkern als ein vor dem Kriege auoh im feindlichen Auslande stets neidlos anerkanntes Vorbild gegolten hat, ein Urteil, das auch heute noch seine volle Bereohtigung besitzt.

Wir werden uns zweifellos auch nach dem Kriege mit den Fragen des chemisohen Unterriohts auf den Schulen und Hochsohulen eingehend befassen müssen. Dabei wird es vor allem auch erforderlich sein, darauf hinzuarbeiten, daB die jetzt leider noch verhaltnismäbig losen Beziehungenzwischen den a kademischen Lehrern und den Schulpada. gogen im engeren Sinnefestergeknüpftwerd e $\mathrm{n}$, denn nur duroh eine gemeinsame Arbeit wird es sohließlich gelingen können, die einer notwendigen Erweiterung des naturwissensohaftlichen und speziell des chemischen Unterrichts entgegenstehenden Schwierigkeiten im allgemeinen Interesse zu überwinden.

[A. 96.]

\section{Glycerinbestimmung nach dem Jodidverfahren mit kleinen Mengen Jodwasserstoffsäure (Halb- mikrojodidverfahren).}

\author{
Von R. NHUMare.
}

(Mitteilung aus dem chemischen Laboratorium des Apollowerkes Wien-Simmering.)

Die quantitative Bestimmung des Glycerins nach dem Verf. von $\mathrm{Z}$ e i s e $\mathrm{l}$ - F a $\mathrm{n} \mathrm{t}^{1}$ ) konnte sich trotz mancher Vorzüge gegenüber anderen Glycerinbestimmungsmethoden keinen allgemeinen Eingang in die chemische Praxis verschaffen. Es wurde ihr vielfach die verhältnismäßig lange Ausführungsdauer und insbesonders der hohe Preis der in Betracht kommenden Reagenzien zum Vorwurfe gemacht, und man fand sie daher als technische Untersuchungsmethode ungeeignet. In den gegenwärtigen Kriegszeiten kommt neben den noch höheren Kosten der Chemikalien auch die schwere Beschaffungsmöglichkeit der Jodwasserstoffsäure hinzu, was ein konstantes Arbeiten nach dem Jodidverfahren völlig unmöglich macht.

Die Bestrebungen gingen infolgedessen dahin, den Verbrauoh an Jodwasserstoffsäure wesentlich zu verringern, und es war der Gedanke naheliegend, durch Umgestaltung der Jodidmethode in ein Mikroverf. zum Ziele zu gelangen. Eine Mikroanalyse im wahrsten Sinne des Wortes wäre für technische $Z$ wecke selbstverständlich undenkbar, da unseren Betriebs- und Untersuchungslaboratorien wohl sohwerlich eine Mikrowage zur Verfügung steht, ja in den meisten Fällen die Unterbringung einer solchen infolge Mangel an geeigneten Räumlichkeiten ganz unmöglich wäre. Es blieb somit zur Lösung dieser Aufgabe nur der Weg, bei möglichster Herabsetzung der Jodwasserstoffmenge die Konzentration der zu untersuchenden glycerinhaltigen Substanz derart zu wählen, daB das Gewicht des gebildeten Jodsilbers auf einer gewöhnlichen, guten, analytischen Wage wie sie sich in jedem besser eingerichteten chemischen Laboratorium vorfindet - festgestellt werden kann, um daraus mit genügender Genauigkeit den Glyceringehalt zu ermitteln.

Fine Anzahl im hiesigen Laboratorium ausgeführter Versuche hat gezeigt, da $B$ das Jodidverfahren bei Benutzung beliebig geringerer Mengen der Probesubstanz und entsprechend herabgesetzter Quantitäten der Reagentien ebenso gut verwendbar ist wie bei genauer Finhaltung der von $\mathrm{Z}$ e is el-Fanto angegebenen Arbeitsweise. So wurden zum Beispiel statt der vorgeschriebenen $5 \mathrm{com}$ bloB 2 com verschiedenster Glycerinlösungen mit nur 6 (statt 15) ccm Jodwasserstoffsüure (D. 1,98) im S t r i t a r schen Jodidapparate ${ }^{2}$ ) dem Jodidverfahren unterworfen (wobei gleichzeitig auch die entsprechend geringere Menge alkoholische Silbernitratlösung - $18 \mathrm{ccm}$ - verwendet wurde). In allen diesen Fällen erhielt man vollständig zufriedenstellende Resultate; eine wesentliche Abkürzung der Arbeitszeit wurde dadurch nicht erzielt.

Weit ökonomischer und vorteilhafter hingegen ist es, zur Jodidbestimmung nur den 10. Teil der Probelösung und der Reagenzien zu verwenden und deren Durchführung in einem eigens für diesen Zweck in verkleinertem Maßstabe angefertigten $S t r i t$ a $r$ schen Jodidapparate vorzunehmen. Dadurch wird nicht nur eine große Ersparnis an Material, sondern auch ein wesentlicher Gewinn an Zeit erzielt, was eine bedeutende Verbilligung der Methode darstellt.

1. De r A p para t. Der Apparat zur Ausführung der im nachfolgenden geschilderten "Halbmikrojodidbestimmung " ist genau so beschaffen wie der von $S$ trit a r konstruierte Jodidapparat, nur wurde er für den vorliegenden Zweck in den halben linearen Dimen. sionen hergestellt, so daB der Rauminhalt des Siedekölbchens und der Voriagen etwa den 8. Teil beträgt (siehe Abb. 1) ${ }^{3}$ ).

In besonderen Fällen ist es vorteilhaft, zwischen den Teilen $b$ und $c$ ein Absorptionsgefä $B g$ in Gestalt eines $S$ t $r$ i t a r schen Kaliappa-

1) Z. f. landw. Versuchswesen in Österr. 5, 729 [1902]; Z. f. analyt. Chemie 42, 549, 579 [1903]; Angew. Chem. 16, 413 [1903].

2) Z. anal. Chem. 42, 579 [1903]

s) Der Apparat ist gesetzlich geschützt und wird von der Firma $P$ a u l Ha a ck, Wien, IX. Garelligasse 4. hergestellt. 